\title{
Distinct Polymer Architecture Mediates Switching of Complement Activation Pathways at the Nanosphere-Serum Interface: Implications for Stealth Nanoparticle Engineering
}

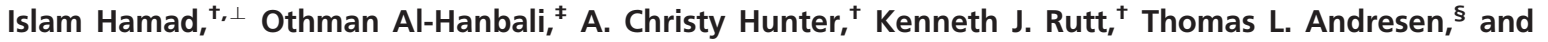 \\ S. Moein Moghimi ${ }^{\ddagger}, *$ \\ ${ }^{\dagger}$ Molecular Targeting and Polymer Toxicology Group, School of Pharmacy, University of Brighton, Brighton BN2 4GJ, U.K., ${ }^{\ddagger}$ Centre for Pharmaceutical Nanotechnology and \\ Nanotoxicology, Faculty of Pharmaceutical Sciences, University of Copenhagen, Universitetsparken 2, DK-2100 Copenhagen Ø, Denmark, and ${ }^{\S}$ Department of Micro- and \\ Nanotechnology, DTU-Nanotech, Technical University of Denmark, Frederiksborgvej, DK-4000 Roskilde, Denmark. ${ }^{\perp}$ Present Address: Department of Pharmaceutical \\ Sciences and Pharmaceutics, Applied Sciences University, Amman, Jordan.
}

S urface camouflaging of nanospheres with poly(ethylene glycol) (PEG) and block copolymers such as poloxamine 908 [a tetrafunctional polyethylene oxide (PEO) - polypropylene oxide (PPO) ethylenediamine block copolymer] is believed to circumvent the bodys defenses (notably against hepatic macrophage, Kupffer cells, recognition, and clearance) thus conferring longevity to nanospheres in the blood following intravenous adminstration. ${ }^{1-3}$ However, the macrophage evading property and circulation times of such engineered nanoparticles depend on their size, rigidity, and surface density of the camouflaging polymers. ${ }^{2,45}$ For example, intravenously injected uncoated and poloxamine 908coated model polystyrene nanoparticles of $50-200 \mathrm{~nm}$ in diameter with PEO chains in the "mushroom" configuration are prone to rapid sequestration by Kupffer cells. In contrast, polystyrene particles of similar size ranges but with surface-projected PEO segments displaying a transitional "mushroom-brush" or "brush" configuration are resistant to rapid Kupffer cell clearance and remain in the blood for prolonged periods. ${ }^{4-6}$ With larger particles $(200-300$ $\mathrm{nm}$ ) displaying mushroom-brush or brush PEO chains splenic clearance

predominates. ${ }^{78}$ The splenic filtration process is a function of particle size and rigidity, since these particles are larger than the width of interendothelial cell slits at the walls of splenic venous sinuses (as in the
ABSTRACT Nanoparticles with surface projected polyethyleneoxide (PE0) chains in "mushroom - brush" and "brush" configurations display stealth properties in systemic circulation and have numerous applications in sitespecific targeting for controlled drug delivery and release as well as diagnostic imaging. We report on the "structure-activity" relationship pertaining to surface-immobilized PEO of various configurations on model nanoparticles, and the initiation of complement cascade, which is the most ancient component of innate human immunity, and its activation may induce clinically significant adverse reactions in some individuals. Conformational states of surface-projected PE0 chains, arising from the block copolymer poloxamine 908 adsorption, on polystyrene nanoparticles trigger complement activation differently. Alteration of copolymer architecture on nanospheres from mushroom to brush configuration not only switches complement activation from C1q-dependent classical to lectin pathway but also reduces the level of generated complement activation products $\mathrm{C} 4 \mathrm{~d}, \mathrm{Bb}, \mathrm{C5a}$, and $\mathrm{SC} 5 \mathrm{~b}-9$. Also, changes in adsorbed polymer configuration trigger alternative pathway activation differently and through different initiators. Notably, the role for properdin-mediated activation of alternative pathway was only restricted to particles displaying PEO chains in a transition mushroom - brush configuration. Since nanoparticle-mediated complement activation is of clinical concern, our findings provide a rational basis for improved surface engineering and design of immunologically safer stealth and targetable nanosystems with polymers for use in clinical medicine.

KEYWORDS: block copolymers - complement activation - nanomedicine poloxamine 908 - stealth nanoparticles

rat model) and their rigidity prevents them from squeezing out in to the venous circulation. ${ }^{7,9}$ Filtered particles are eventually taken up by the splenic red-pulp macrophages presumably as a result of partial displacement of the adsorbed copolymer in the spleen red-pulp. For particles above $300 \mathrm{~nm}$ in size the poloxamine coating is ineffective (even in brush conformation) in combating rapid Kupffer cell clearance. This probably indicates that the

\begin{abstract}
*Address correspondence to momo@farma.ku.dk.
\end{abstract}

Received for review August 11, 2010 and accepted October 18, 2010.

Published online October 28, 2010. 10.1021/nn101990a

() 2010 American Chemical Society 


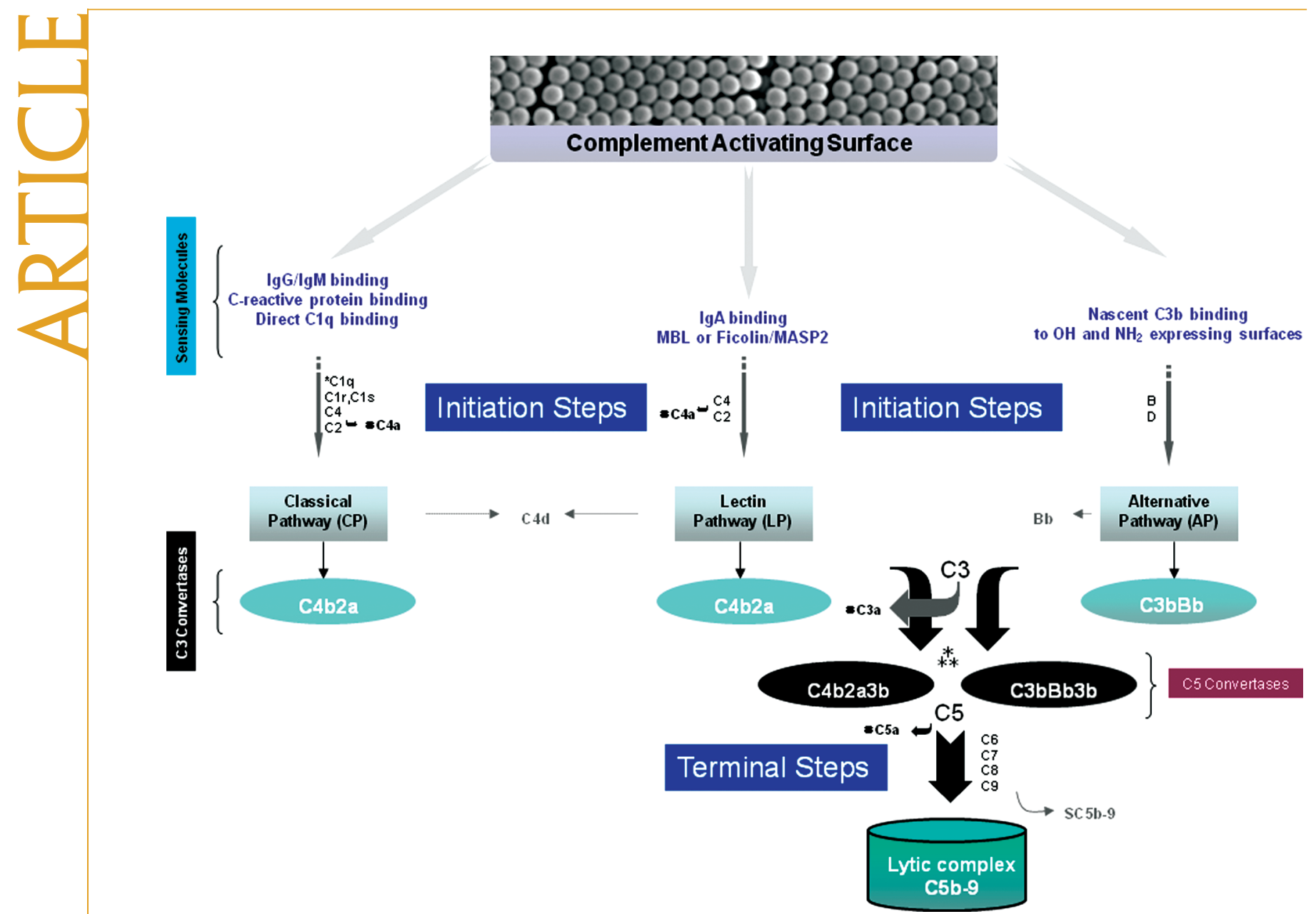

Figure 1. Complement activation pathways. Surface binding of sensing molecules triggers complement through different pathways. The classical pathway is initiated by the binding of the C1q component of the C1 protein to native or antibody or C-reactive proteindecorated surfaces. The lectin pathway is typically activated when mannose-binding lectin (MBL) or ficolin interact with surface sugar molecules (mannose) or some other surface entities. Sequential activation of other complement proteins for each pathway is shown; however, activation of both pathways leads to the assembly of C3 convertase C4b2a. Direct cleavage of C3 by MBL-associated serine proteases can also occur. The alternative pathway is a self-amplification loop driven by $\mathrm{C} 3 \mathrm{bBb}$ convertase. Alternative pathway turnover can occur secondary to classical and lectin pathway activation or be initiated directly by foreign surfaces. The later occurs through low level spontaneous C3 "tickover" generating nascent C3b, which is susceptible to nucleophilic attack by $\mathrm{OH}_{\text {or }} \mathrm{NH}_{2} \mathrm{groups}$ exposed on nanoparticle surfaces. Direct C3 binding may also trigger an alternative pathway. The terminal step of complement pathways generates $\mathrm{C} 5$ convertases that through sequential activation of complement proteins $\mathrm{C} 5$ to $\mathrm{C} 9$ assemble the terminal $\mathrm{C} 5 \mathrm{~b}-9$ complement membrane-attack complex.

dimensions of the stabilizing chains of the copolymer does not exceed the range of van der Waals attraction force, thus making particles susceptible to blood opsonization processes and hence macrophage recognition and clearance.

Complement is an ancient component of innate immunity that monitors host invaders including manmade nanoparticles. ${ }^{10,11}$ It comprises three distinct pathways (classical, lectin, and alternative), and is functionally composed of over 30 soluble and membranebound proteins, with each responding to a different set of activators (Figure 1). Complement primes targets (including stealth nanosystems) ${ }^{1,2}$ for immune clearance through surface opsonization with C3 cleavage products $\mathrm{C} 3 \mathrm{~b}$ and $\mathrm{iC} 3 \mathrm{~b}$, triggers inflammatory reactions, and may induce tissue damage (e.g., directly by the lytic C5b-9 complex) and initiate adverse reactions (e.g., indirectly by anaphylatoxins $\mathrm{C} 3 \mathrm{a}$ and $\mathrm{C} 5 \mathrm{a}$ through their ef- fect on different blood cells) in some individuals. ${ }^{10,12-15}$ Complement also guides the adaptive immune response. There are clinical reports of acute hypersensitivity reactions to infusion of long circulating regulatoryapproved nanoparticles (e.g., Doxil, a PEGylated liposome with entrapped doxorubicin) in certain individuals. ${ }^{16-18}$ These reactions are non-IgE mediated and often associated with flushing and circulatory/cardiac disturbances. ${ }^{19}$ Neuro-psychosmatic and vegetative responses have further been observed following Doxil infusion in dogs. ${ }^{19}$ Recent studies in pigs have suggested that the cardiopulmonary distress caused by Doxil and other nanoparticles strongly correlates with complement activation..$^{20}$ It is now known that PEGylated liposomes can trigger complement activation in the absence of anti-PEG antibodies through both C1q-dependent classical and alternative pathways. ${ }^{21}$ The incidence of such adverse responses corresponds 
(a)

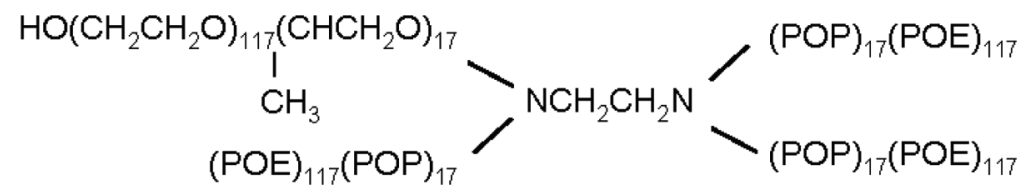

(b)

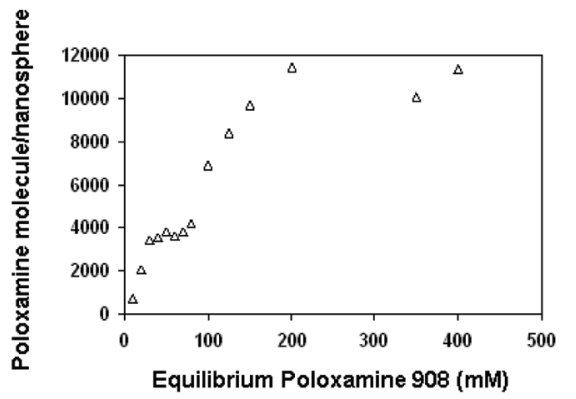

(c)

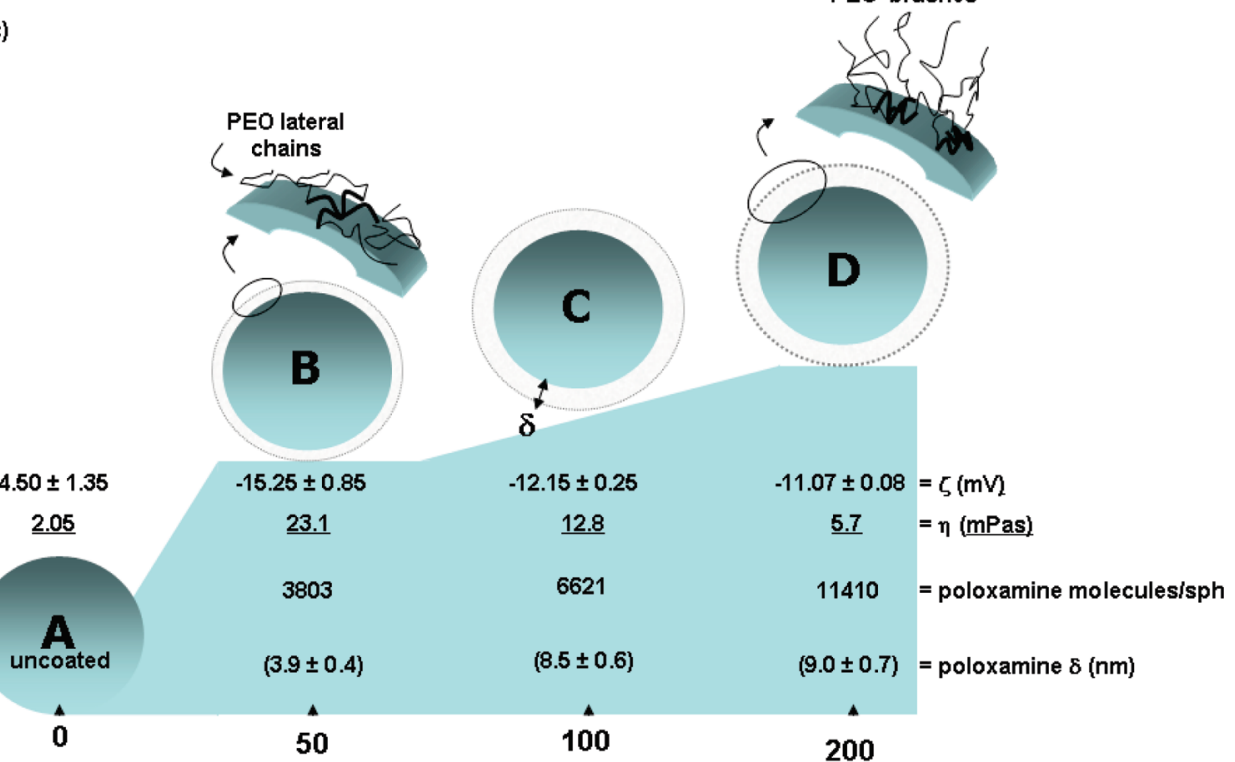

Equilibrium poloxamine $908(\mu \mathrm{g} / \mathrm{mL})$

(d)
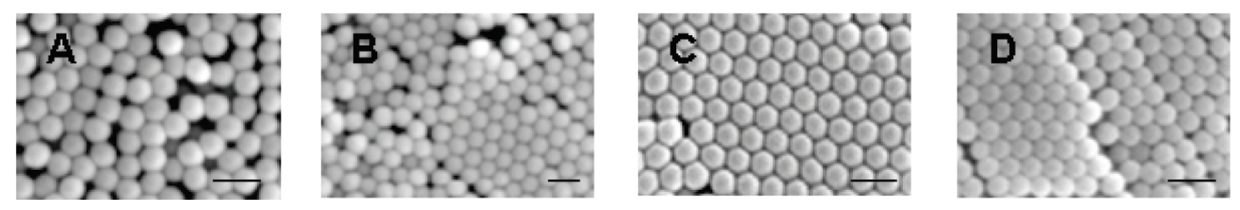

Figure 2. Physicochemical characteristics of uncoated and poloxamine 908-conditioned polystyrene nanoparticles. (a) Poloxamine 908 structure, (b) a single curve poloxamine 908 adsorption isotherm with a bimodal feature, (c) physicochemical characteristics of poloxamine-coated nanoparticles from selected regions of the adsorption isotherm, and (d) scanning electron micrographs of representative nanoparticles on a graphite base; bar $=500 \mathrm{~nm}$.

to approximately $80 \%$ of all immune-mediated hypersensitivity reactions; this translates to the occurrence of at least 400000 severe reactions with 20000 fatalities every year in the United States only. ${ }^{22}$ Such outcomes can therefore complicate the development phase of new nanomedicines and other potential multifunctional/composite nanoparticles for clinical applications. In this respect, previous attempts have rarely focused in assessing the role of surface projected polymers on pathways and mechanisms of complement activation. These issues are also important in terms of optimizing nanoparticulate drug carrier stability (particularly for vesicular systems, since complement activation can damage the liposomal bilayer resulting in premature drug leakage) and pharmacokinetics in the blood, as well as for translation in to surface engineering of other nanomaterials of different morphology and structure (e.g., carbon nanotubes) for improving their biocompatibility. ${ }^{23}$

Since block copolymers of poloxamer and poloxamine series are widely used for nanoparticle surface engineering, ${ }^{1,4,6-9,24-27}$ we have investigated and compared complement activation in human serum by poloxamine 908-coated nanoparticles of $230 \mathrm{~nm}$ with 


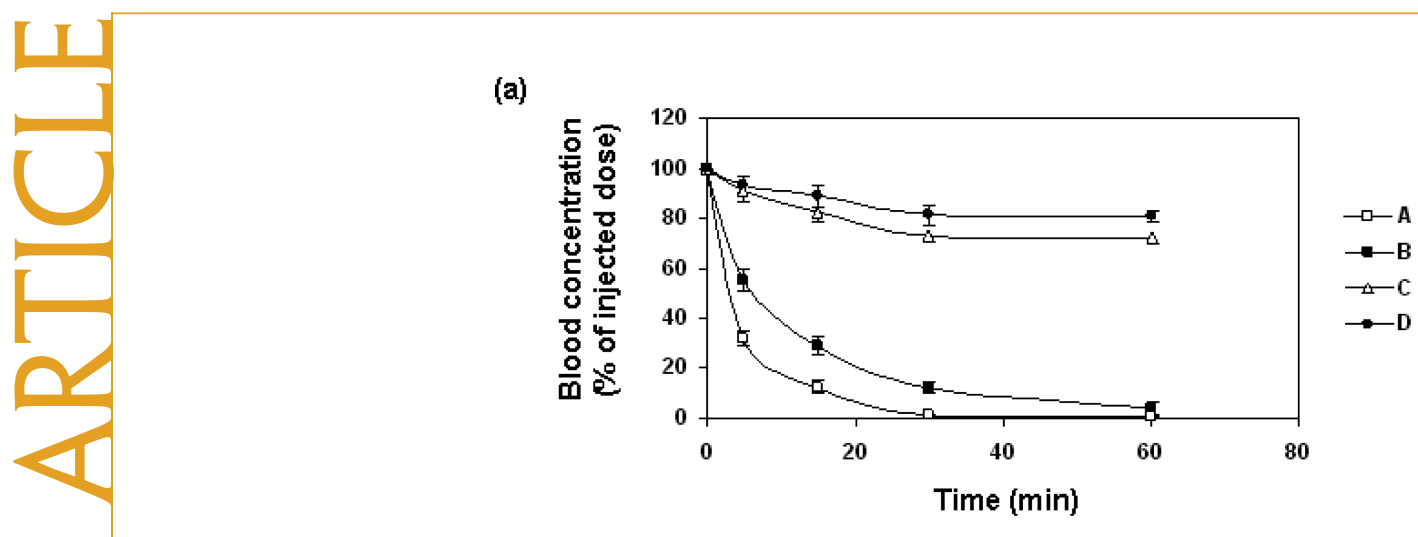

(b)

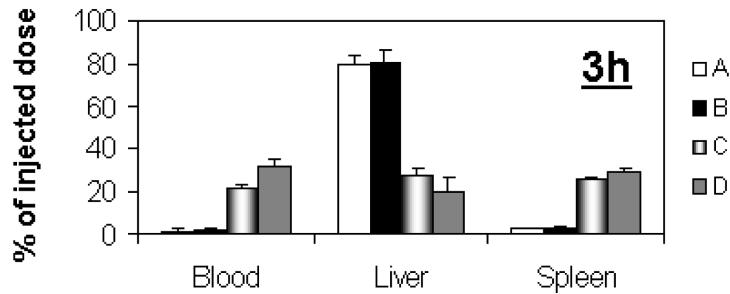

(c)

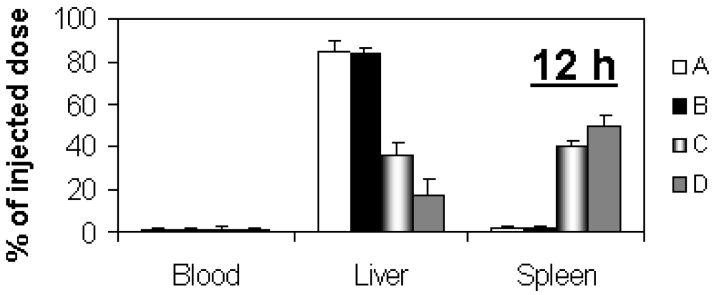

Figure 3. Blood clearance and organ distribution of intravenously injected poloxamine-coated nanospheres. Uncoated nanospheres are designated as A; designations B, C, and D refer to poloxamine-coated nanospheres from the 1st plateau, midway, and top plateau of the adsorption isotherm, respectively.

surface-projected PEO chains displaying mushroom, mushroom - brush, and brush configurations. The physicochemical properties of poloxamine-coated particles are well characterized and established, ${ }^{5}$ therefore they serve as an excellent working platform for assessing structure-activity relationships to aid in the understanding of key mechanistic issues pertaining to complement and innate immunity. We now demonstrate that poloxamine 908-coated nanospheres incite complement activation in human serum regardless of PEO chain configuration, but remarkably alteration of copolymer architecture on nanospheres from mushroom to mushroom - brush configuration switches activation from the classical to the lectin pathway. Also, variation in adsorbed polymer configuration triggers alternative pathway activation differently and the mechanistic aspects are discussed. Since nanoparticlemediated complement activation is of significant clinical concern, our findings provide a rational basis for precision surface engineering and design of immunologically safer stealth and target-specific nanosystems with polymers for application in clinical medicine.

\section{RESULTS AND DISCUSSION}

Nanoparticle Characteristics. In accordance with our previous study ${ }^{5}$ the results in Figure $2 \mathrm{~b}$ demonstrate a bimodal pattern for poloxamine 908 adsorption onto the surface of near monodispersed polystyrene nanoparticles $(230 \mathrm{~nm})$. This feature reflects dynamic changes in the arrangement of surface projected polyethylene oxide (PEO) chains; a flat or mushroom-like conformation at the first plateau region of the isotherm, followed by a transition into a brush-like conformation, as previously assessed by us using a combination of laser light scattering, viscosity measurements, and nanorheological studies with atomic force microscopy (height and phase lag measurements in tapping mode). ${ }^{5}$ For structure-activity studies in relation to nanosphere surface properties and initiation of complement activation we explicitly focused on representative nanospheres from each plateau region of the adsorption isotherm ( $B$ and $D)$, and a population between the plateau regions $(C)$, and compared them with uncoated nanospheres (A), Figure 2c. Physicochemical characteristics of the selected nanospheres [poloxamine adsorbed layer thickness $(\delta)$, poloxamine surface density, zeta potential $(\zeta)$, and apparent viscosity] are presented in Figure 2c; and the results are comparable with our previous study. ${ }^{5}$ SEM micrographs further demonstrate the effect of polymer conformation on the packing arrangement of nanoparticles (Figure 2c). Uncoated nanospheres aggregate in an unstructured way, but form regular arrangements when coated with poloxamine 908 to the level of the first plateau of the adsorption isotherm, where the available surface area is in the region of $45 \mathrm{~nm}^{2} /$ poloxamine molecule. For types $C$ and $D$ nanoparticles the coating arrangement translates to a surface area of 30 and $15 \mathrm{~nm}^{2} /$ poloxamine molecule, respectively, where subsequent layers form a three-dimensional close-packed array (as in type D). This presumably arises from the repulsive forces of ether oxygen atoms of projecting PEO chains.

Biodistribution Studies. The results in Figure 3 demonstrate differences in blood clearance profile and organ distribution of engineered nanopsheres, which are in line with the presumed surface-projected PEO configurational states. Accordingly, nanospheres $A$ and $B$ are rapidly cleared by the hepatic macrophages, whereas 
(a)

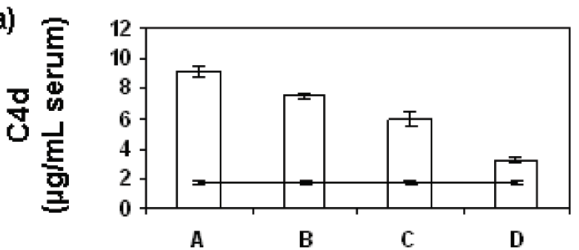

(b)
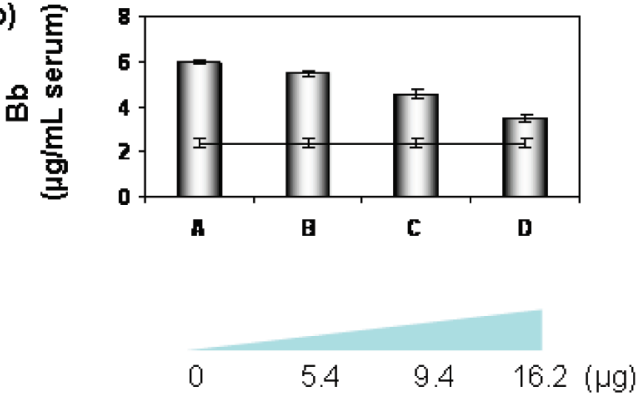

Surface bound poloxaminelincubation

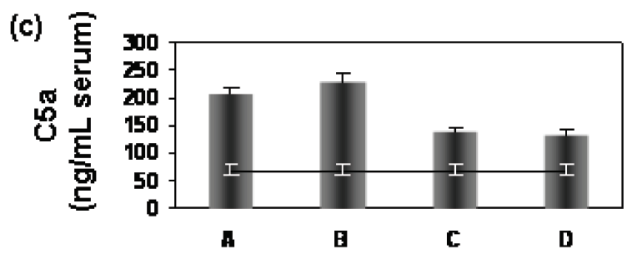

(d)

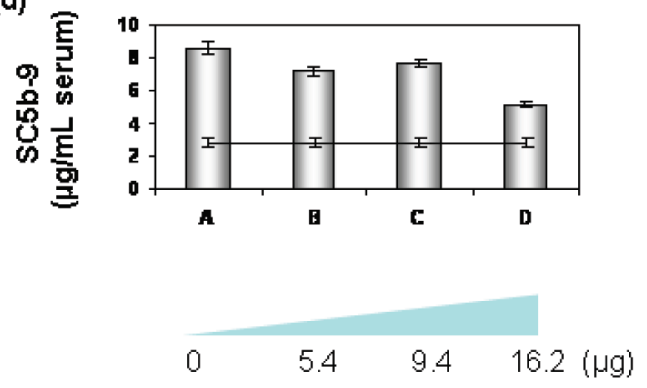

Surface bound poloxaminelincubation

Figure 4. Nanosphere-mediated elevation of complement activation products in human serum. The horizontal lines $(a-d)$ denote background levels of activation products.

nanospheres $C$ and $D$ exhibit stealth properties but are eventually filtered by the spleen.

Complement Activation by Nanoparticles. Uncoated nanospheres dramatically elevated serum levels of fluidphase complement activation products $\mathrm{C} 4 \mathrm{~d}$ (a degradation product of $\mathrm{C} 4$, mediated by complement control protein C4bp and factor I, and an established marker of both classical and lectin pathways), ${ }^{21,28} \mathrm{Bb}$ (an established marker of the alternative pathway turnover resulting from Factor B cleavage), ${ }^{21,28} \mathrm{C} 5$ a (the most potent complement anaphylatoxin), and the nonlytic SC5b-9 (the vitronectin or S protein bound form of C5b-9, and a marker of terminal complement pathway) ${ }^{21}$ above background (Figure 4). Poloxamine coating did not abrogate complement activation fully. Complement activation occurred with both nanospheres B (displaying mushroom PEO conformation) and $C$ (with a transitional mushroom-brush configuration), but dramatically reduced with type $D$ entities, where the surface projected PEO chains predominantly assume brush-like arrangement.

The Role of Classical and Lectin Pathways of Complement. Surface binding of C1q component of the complement protein $\mathrm{C} 1$ complex triggers activation of the classical pathway of complement. ${ }^{10,21}$ This mode of activation generates the $\mathrm{C} 3$ convertase $\mathrm{C} 4 \mathrm{~b} 2 \mathrm{a}$ through sequential activation of $C 4$ and $C 2$ proteins involving serine proteases $\mathrm{C} 1 \mathrm{r}$ and $\mathrm{C} 1 \mathrm{~s}$ (Figure 1). To distinguish between nanoparticle-mediated activation of classical and lectin pathways of complement, serum was immunochemically depleted from C1q, and C4d levels were monitored in C1q-depleted serum. This distinguishes between the classical and lectin pathways, since the latter is activated through surface binding of mannose lectin-binding protein (MBL) and/or ficolins. The binding of MBL and/or ficolins to nanoparticles in turn induces activation of the MBL-associated serine proteases (MASPs), notably MASP-2. This subsequently cleaves the $C 4$ protein resulting in ultimate formation of $C 4 \mathrm{~b} 2 \mathrm{a}$ convertase, which structurally is identical to the classical pathway C3 convertase. ${ }^{28-30}$

Uncoated nanospheres did not affect C4d levels in C1q-depleted serum, but these increased dramatically when physiological concentrations of $\mathrm{C} 1 \mathrm{q}$ was added (Table 1). This attests to the involvement of C1qmediated classical pathway activation. Similar results were obtained with type B nanospheres, but not with nanospheres $C$ and $D$ where activation is predominantly via lectin pathway, since the nanosphere challenge dramatically elevates C4d levels in C1q-depleted serum

TABLE 1. Nanoparticle-Mediated C4d Rises in C1q-Depleted Human Serum

\begin{tabular}{|c|c|c|c|c|}
\hline \multirow[b]{2}{*}{ nanospheres } & \multicolumn{4}{|c|}{ C $4 \mathrm{~d}(\mu \mathrm{g} / \mathrm{mL}$ serum) } \\
\hline & $-C_{1 q}$ treatment & $+\mathrm{C} 1 q$ treatment & + nonspecific Ab treatment & + anti-MASP2 Ab treatment \\
\hline none & $6.2 \pm 0.8$ & $7.4 \pm 0.9$ & $7.3 \pm 1.0$ & $7.5 \pm 0.4$ \\
\hline A (uncoated) & $6.8 \pm 0.6$ & $22.6 \pm 1.1$ & $7.1 \pm 0.4$ & $8.3 \pm 0.5$ \\
\hline B & $5.6 \pm 0.9$ & $18.9 \pm 0.7$ & $6.1 \pm 0.9$ & $8.8 \pm 1.4$ \\
\hline$C$ & $15.2 \pm 1.2$ & $18.2 \pm 0.8$ & $17.5 \pm 1.6$ & $8.8 \pm 1.9$ \\
\hline$D$ & $11.8 \pm 0.6$ & $12.9 \pm 0.8$ & $12.8 \pm 1.1$ & $6.9 \pm 1.8$ \\
\hline
\end{tabular}



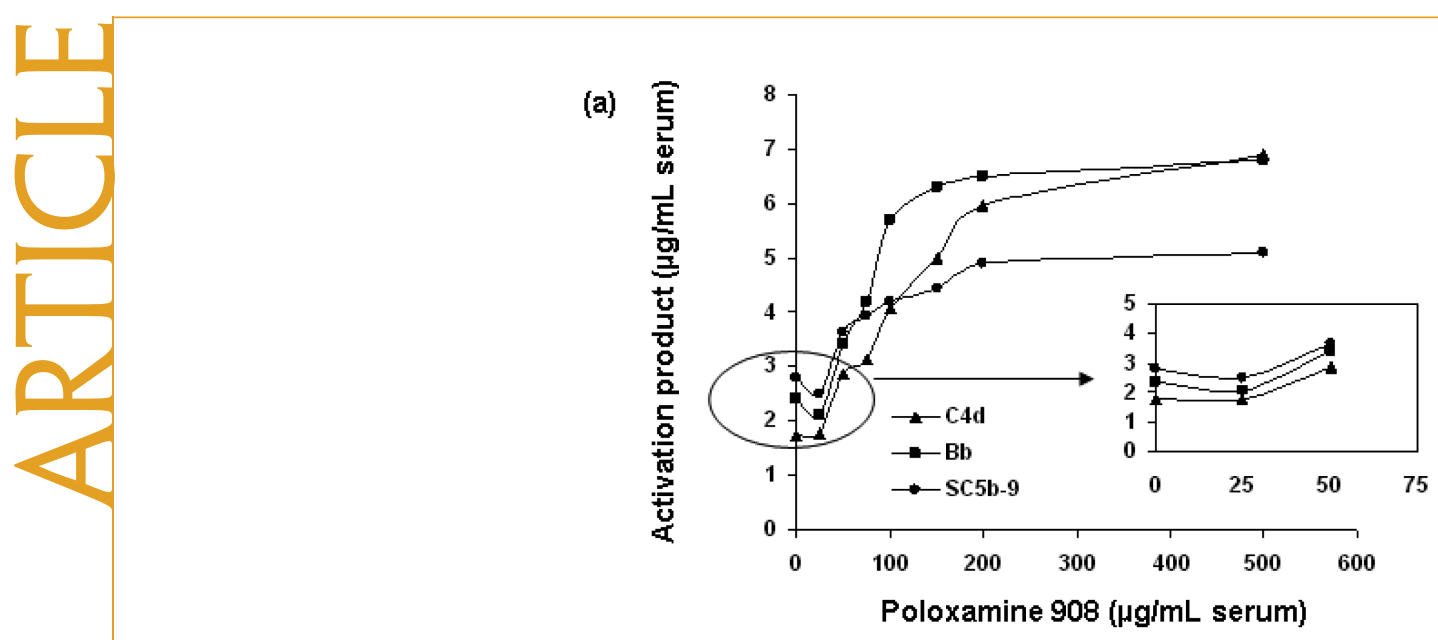

(b)

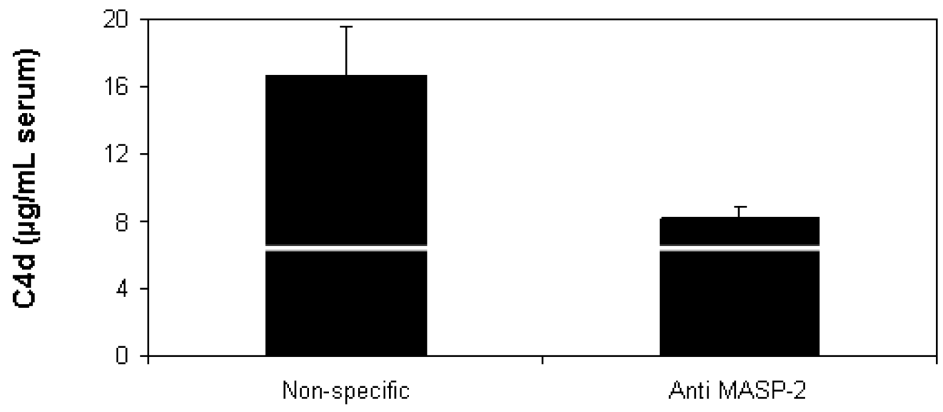

Antibody

Figure 5. Poloxamine 908-mediated complement activation in normal human serum (a) and poloxamine-mediated C4d elevation in C1q-depleted human serum. In panel b poloxamine concentration was $100 \mu \mathrm{g} / \mathrm{mL}$ serum. C4d elevation is abrogated in the presence of anti MASP-2 antibodies indicating a role for the lectin pathway in C4 cleavage. A nonspecific IgG was included in the control experiment. The white line indicates the background level of serum C4d in the presence of nonspecific antibody. Poloxamine samples may contain impurities introduced during synthesis and may also contain small amounts of butylated hydroxytoluene to prevent degradation. Earlier we demonstrated that such impurities and the presence of antioxidants have no effect on complement activation. ${ }^{16}$ Complement activation by poloxamer and poloxamine is an intrinsic property of these macromolecules and is independent of the degree of sample polydispersity. ${ }^{16}$

(Table 1). This was further confirmed by blocking the activity of the lectin pathway convertase MASP-2 by an anti-MASP-2 monoclonal antibody (Table 1).

With type B nanospheres, incubations contain 5.4 $\mu \mathrm{g}$ of surface-bound poloxamine in a final volume of $40 \mu \mathrm{L}$ serum; this translates to an equivalent of $135 \mu \mathrm{g}$ of free poloxamine/mL serum, which is below its $\mathrm{cmc}$ value $(700 \mu \mathrm{g} / \mathrm{mL})$ determined by both static light scattering and fluorescence anisotropy. Free poloxamine at concentrations above $75 \mu \mathrm{g} / \mathrm{mL}$ serum dramatically elevate serum C4d levels (Figure 5a); this is exclusively lectin pathway driven, since C4d elevation is blocked by anti MASP-2 antibodies (Figure 5b). If surfaceadsorbed poloxamine molecules were displaced by serum proteins, then poloxamine molecules should have further triggered complement in C1q-depleted serum via lectin pathway. Therefore, C1q-dependent complement activation by nanosphere $B$ is a true reflection of surface property, where a mushroom-like PEO configuration neither triggers lectin pathway nor blocks $\mathrm{C} 1 \mathrm{q}$ binding to the nanosphere surface by steric hindrance. This further reflects the role of PEO conformation in differential binding of key serum/plasma protein, at least in relation to complement components.
Lectin pathway activation by nanospheres $C$ and $D$ is remarkable (Table 1), since surface-projected PEO chains were expected to dramatically suppress surface opsonization events. ${ }^{2}$ It is unlikely that the lectin pathway is triggered by displaced (free) poloxamine molecules. Earlier work with radio-labeled poloxamine 908coated (at plateau region of the adsorption isotherm) polystyrene nanoparticles $(250 \mathrm{~nm})$ demonstrated that not more than $10 \%$ of poloxamine molecules are released after $3 \mathrm{~h}$ incubation in serum. ${ }^{31}$ With nanospheres $C$ and $D$, this translates to less than 25 and 40 $\mu \mathrm{g}$ poloxamine $/ \mathrm{mL}$ serum, respectively, which is far below the minimum free poloxamine concentration necessary to trigger complement via the lectin pathway (Figure 5a). Therefore, nanosphere-mediated lectin pathway activation is likely due to interaction between MBL and/or ficolin with projected PEO chains, resulting in activation of their associated serine protease zymogen MASP-2. Indeed, nanosphere-mediated C4d elevation in serum is blocked in the presence of $\mathrm{N}$-acetylglucosamine, a substrate for both MBL and ficolin (not shown). ${ }^{28}$ It is interesting to note that there are structural similarities between the terminal PEO chains of poloxamine and a region of D-mannose that 
(a)

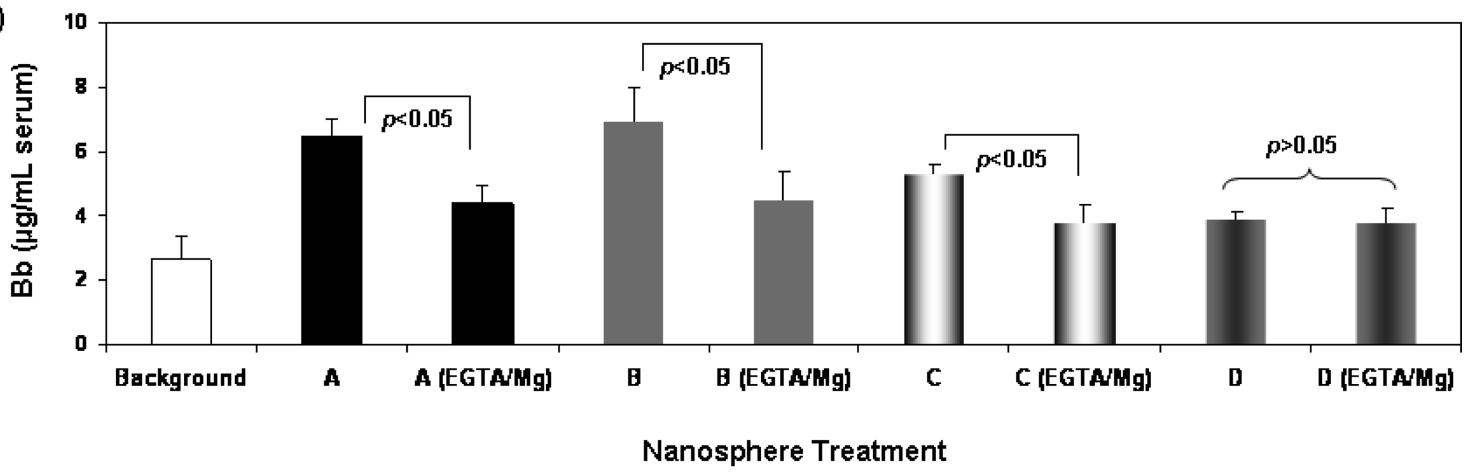

(b)

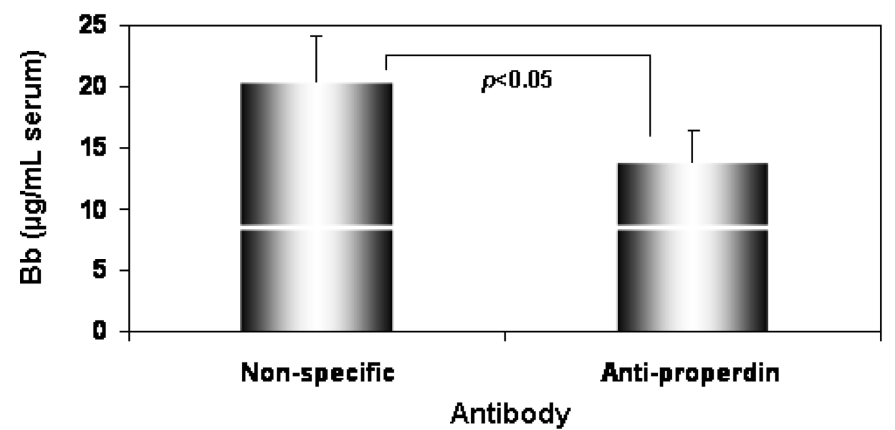

Figure 6. Nanosphere-mediated triggering of Bb elevation in the presence of $\mathrm{EGTA} / \mathrm{Mg}^{2+}(\mathrm{a})$ and the role of properdin in nanosphere-mediated Bb elevation in C1q-depleted human serum with functional lectin pathway (b). In panel b the effect was exclusive with type $\mathrm{C}$ nanospheres. The white line indicates background $\mathrm{Bb}$ level in serum in the presence of the nonspecific antibody.

could trigger lectin pathway activation at a certain surface density. ${ }^{22}$

Increasing surface density of poloxamine (as in nanosphere D) significantly reduces $\mathrm{C} 4 \mathrm{~d}$ and SC5b-9 generation compared with type $C$ nanospheres, but C4d elevation is still through the lectin pathway (Figure 4). Therefore, denser surface PEO brushes (with decreased mobility) ${ }^{32}$ may sterically minimize MBL/ficolinMASP-2 binding to PEO backbone or to surface accumulated mannosylated glycoproteins, such as various apolipoproteins. ${ }^{25}$

The Role of Alternative Pathway. The alternative pathway of complement is stimulated by the spontaneous cleavage of the thioester bond in C3 ("C3-tickover") or when the internal thioester bond in the $\alpha$-chain of nascent $\mathrm{C} 3 \mathrm{~b}$ undergoes nucleophilic attack in the presence of structures rich in nucleophilic groups (e.g., hydroxyl-rich surfaces). ${ }^{10,28,33}$ Nanosphere-mediated elevation of alternative pathway turnover, as evident from $\mathrm{Bb}$ rises above background ${ }^{13}$ (Figure $4 \mathrm{~b}$ ), is either secondary to classical and lectin pathway activation (amplification loop) or is initiated independently (e.g., C3 tickover and nascent C3b binding). ${ }^{10}$ Both routes are operative with all types of nanospheres, with the exception of type $\mathrm{D}$, since nanosphere-mediated $\mathrm{Bb}$ elevation is reduced by approximately $30 \%$ in the presence of EGTA/ $\mathrm{Mg}^{2+}$ (activation of classical and lectin path-

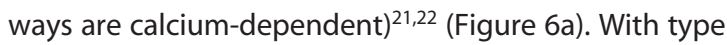
D nanospheres complement activation is exclusively through the amplification loop since no Bb elevation

www.acsnano.org above background level was detected in the presence of EGTA/ $\mathrm{Mg}^{2+}$, Figure 6a. Interestingly, type $\mathrm{C}$ particles were able to increase alternative pathway turnover through interaction with properdin (a positive regulator as well as a recently identified initiator of alternative pathway) ${ }^{34,35}$ as demonstrated in reduction of $\mathrm{Bb}$ levels in the presence of antiproperdin antibodies (Figure 6b). This is presumably due to noncovalent binding of properdin to poloxamine chains, thereby recruiting $\mathrm{C} 3 \mathrm{~b}$ and allowing for alternative pathway convertase build-up. Therefore, for the first time we have demonstrated that poloxamine surface configuration affects alternative pathway activation through different processes.

\section{CONCLUSION}

We have demonstrated the importance of configurational/mobility of surface projected PEO chains in modulation of complement activation with a spectrum of model nanoparticles that exhibit different pharmacokinetic profiles. Although stealth properties, and hence long circulatory profiles, can be achieved by partial poloxamine surface coverage, immunologically it is necessary to maximize coating. This dramatically reduces complement activation, presumably due to decrease in PEO chain mobility, ${ }^{1,32}$ and the risk of adverse infusion reactions. ${ }^{1,14,23}$ In sensitive individuals, rapid production of complement anaphylatoxins ( $\mathrm{C} 3 \mathrm{a}$ and $\mathrm{C} 5 \mathrm{a}$ ) initiates the release of a wide spectrum of secondary mediators that subsequently triggers adverse effects. To this end, 
structure-activity relationships between complement activation and conformational states of immobilized copolymers must be considered for rational design of immunologically safe stealth nanoparticles. Particular emphasis must be placed to suppress or prevent complement activation through the alternative pathway, which presents a unique role in the complement activation cascade. Since alternative pathway is activated by its own product (C3b), it functions as a positive feedback loop, producing a vigorous local response. Our observation that properdin binding to type $C$ nanospheres can also trigger alternative pathways is of concern. Properdin is synthesized by blood monocytes, $\mathrm{CD}^{4+}$ and $\mathrm{CD}^{8+}$ blood T cells, and neutrophils. ${ }^{36}$ In neutrophils, properdin is stored in secondary granules and can be released following stimulation with secondary mediators. ${ }^{37}$ In the blood, rapid properdin release, and following binding to surfaces such as type $C$ nanoparticles, may further aid C5a generation through alternative pathway convertase assembly and inflict further damage. This may further disrupt the delicate balance between complement regulation and complement activation. Accordingly, assessment of complement activation pathways by the described nanospheres in whole blood would be of immense interest and should be investigated. Furthermore, future efforts should concentrate in assessing the role of immobilized polymer conformation on particles of $100 \mathrm{~nm}$ or below in size since curvature cannot only control polymer mobility but also modulate surface deposition of sensing molecules (e.g., antibodies, C1q, etc.) that trigger complement. ${ }^{2}$ In addition, smaller sizes $(30 \mathrm{~nm}$ and below) in conjugation with surface dynamic events arising from adsorbed or conjugated polymers may restrict surface assembly of complement convertases, which are of similar size ranges. ${ }^{2}$ Our findings may also be relevant to mechanistic understanding of complement activation by biodegradable polymeric stealth nanoparticles such as PEGylated polyalkylcyanoacrylate nanospheres and PEGylated poly(D,L,lactide) nanocapsules, and could lead to design of safer polymeric stealth systems for clinical applications. Similar to poloxamine 908-coated nanoparticles, these entities activate complement regardless of surface PEG chain length and density, but the extent of complement activation decreases with longer PEG chains. ${ }^{38,39}$ However, mechanistic aspects still remain to be resolved.

Previously we have demonstrated that splenotropic poloxamine-coated nanoparticles following splenic filtration are internalized by splenic red-pulp macrophages. ${ }^{8}$ Although complement fixation and opsonization by $\mathrm{C} 3 \mathrm{~b}$ and $\mathrm{iC} 3 \mathrm{~b}$ could facilitate nanoparticle uptake by these macrophages, recent studies with PEGylated liposomes, which also fix complement opsonic proteins, have shown that the steric barrier of projected PEG interferes with the binding of the opsonic protein to the macrophage complement receptor. ${ }^{21}$ This process is most likely to occur with nanospheres $C$ and $D$, otherwise rapid hepatic sequestration would have occurred. Therefore, alternative splenic red-pulp macrophage uptake mechanisms are in operation with regard to clearance of filtered poloxamine-coated nanoparticles and are worthy of future investigation.

\section{MATERIALS AND METHODS}

Poloxamine Characterization and Adsorption Isotherm Studies. Poloxamine 908 was a kind gift of BASF Aktiengesellschaft (Ludwigshafen, Germany), used without further modification, and characterized as before $\left(M_{\mathrm{w}}=22455 ; M_{\mathrm{n}}=13220 ; \mathrm{Mz}=28190\right) .^{5}$ Endotoxin-free poloxamine 908 solutions were prepared in 10 $\mathrm{mM}$ Mcllvaine buffer ( $\mathrm{pH}$ 7.2). The critical micelle concentration (cmc) of poloxamine 908 was determined by static light scattering. Measurements were performed at $37^{\circ} \mathrm{C}$ at a $90^{\circ}$ angle using a BI-SM200 Brookhaven instrument, equipped with a $100 \mathrm{~mW}$ $\mathrm{He}-\mathrm{Ne}$ laser with a wavelength of $632.8 \mathrm{~nm}$. The sample cell was embedded in a refractive index matched bath. The static light scattering from poloxamine 908 in the concentration range from $0.01-64 \mathrm{mg} / \mathrm{mL}$ was measured. Each measurement was done in triplicate. The $\mathrm{cmc}$ was further measured by fluorescence anisotropy on a Hitachi F7000 fluorescence spectrophotometer giving a comparable value.

Polystyrene nanoparticles ( $2.5 \% \mathrm{w} / \mathrm{v}), 230 \pm 0.5 \mathrm{~nm}$ in diameter, manufactured by Polysciences Inc. (USA), were purchased from Park Scientific (Northampton, UK). Poloxamine-coated particles were prepared in triplicate by adding $1.25 \mathrm{mg}$ of polystyrene nanoparticles (endotoxin free) to poloxamine solutions ranging from 0 to $400 \mu \mathrm{g} / \mathrm{mL}$ poloxamine $(0-0.0178 \mathrm{mM})$ in a total volume of $1.0 \mathrm{~mL}$ in Eppendorf tubes. All tubes were shaken gently $(100 \mathrm{rpm})$ for $24 \mathrm{~h}$ at $25^{\circ} \mathrm{C}$ in a water bath. The suspensions were then centrifuged for $40 \mathrm{~min}$ at $16000 \mathrm{~g}$. No polystyrene particles were lost in supernatants as monitored by light scattering during the centrifugation steps. Poloxamine concentration was determined by a highly sensitive biphasic colorimet- ric assay for the resulting supernatants using a standard curve run concurrently with the adsorption experiment (control tubes without any polystyrene particles but containing the same concentrations of poloxamine as in adsorption isotherm). ${ }^{40}$ Briefly, for the colorimetric assay, aliquots (in a total volume of $0.5 \mathrm{~mL}$ ) of standard poloxamine solutions (in Mcllvaine buffer, $\mathrm{pH}$ 7.2) or supernatants from the adsorption isotherm were added to Eppendorf tubes containing $0.5 \mathrm{~mL}$ of chloroform and $0.5 \mathrm{~mL}$ of reagent solution ( $400 \mathrm{mM}$ ammonium thiocyanate and $100 \mathrm{mM}$ anhydrous ferric chloride). The system was then mixed vigorously for $20 \mathrm{~min}$ at room temperature to attain equilibrium, followed by centrifugation ( $3 \mathrm{~min}$ ) using a microfuge at $16000 \mathrm{~g}$. The lower chloroform layer was extracted, transferred to a quartz cuvette with $1 \mathrm{~cm}$ path length, and covered with a small volume of the reagent solution. The absorbance was measured at $510 \mathrm{~nm}$ using a spectrophotometer.

Particle Size and Electrophoretic Mobility. The hydrodynamic radius and the electrophoretic mobility of particles were determined by photon correlation spectroscopy (PCS) and laser Doppler electrophoresis, respectively, in $10 \mathrm{mM}$ Mcllvaine buffer, $\mathrm{pH}$ 7.2 , at $25^{\circ} \mathrm{C}$ using a Zetasizer 3000 system (Malvern Instruments, UK) as described previously. ${ }^{5,26}$ PCS measurements (based on CONTIN analysis) were determined at a wavelength of 633 $\mathrm{nm}$, scattering angle of $90^{\circ}$, dispersant viscosity of $0.89 \mathrm{CP}$, and refractive index of 1.35, respectively. For electrophoretic mobility measurement conditions were as follows: dielectric constant, 78.3; current, $1.4 \mathrm{~mA}$; fluid refractive index of 1.35; cell field of $29.3 \mathrm{~V} / \mathrm{cm}$; viscosity of $0.89 \mathrm{cP}$; and conductivity of $0.7 \mathrm{mS} / \mathrm{cm}$. 
Apparent Viscosity Measurements. The viscosity of nanoparticle suspensions (volume fraction $\phi=0.18$ ), was measured at $25 \pm$ $0.1^{\circ} \mathrm{C}$ using a Thermo Haake RheoStress 1 rheometer (Gebrüder HAAKE GmbH, Germany). ${ }^{5}$ A C30 cone-plate sensor system with $1^{\circ}$ angle and a gap of $0.054 \mathrm{~mm}$ was used. Poloxaminecoated nanoparticles were free from unbound poloxamine molecules. The viscosity (apparent) was obtained from the slope of linear part (from 8 to $16 \mathrm{~s}^{-1}$ ) of the shear strain versus shear rate plot. An oscillation test was also performed with nanoparticle suspensions at a constant frequency of $1 \mathrm{~Hz}$ and strain of $0.05-20$. Each test was repeated three times. Data analysis was carried out with the Thermo Haake software RheoWin Pro.

Serum Characterization and Assays of in Vitro Complement Activation. To measure complement activation in vitro, we determined nanoparticles/polymer-induced rise of human serum complement activation product $\mathrm{C} 4 \mathrm{~d}, \mathrm{Bb}, \mathrm{C} 5 \mathrm{a}$, and $\mathrm{SC} 5 \mathrm{~b}-9$ using respective Quidels Enzyme-Linked Immunosorbant Assay (ELISA) kits according to the manufacturers protocols as described previously. ${ }^{11,22,28} \mathrm{C} 1 \mathrm{q}$-depleted human serum (immunochemically depleted of (1q) was obtained from Quidel. The complement hemolytic activity of $\mathrm{C} 1 \mathrm{q}$-depleted serum was restorable following the addition of pure $\mathrm{C} 1 \mathrm{q}(180 \mu \mathrm{g} / \mathrm{mL})$ as assessed by the hemolytic test using sheep erythrocytes sensitized with rabbit antisheep erythrocyte antibody. ${ }^{28}$ The functional activity of classical, lectin, and the alternative pathways of complement were confirmed in all sera with Wielisa-Total Complement Screen kit prior to functional studies. ${ }^{28}$

For measurement of complement activation, the reaction was started by adding $250 \mu \mathrm{g}$ of designated nanoparticles (in sterile physiological saline) or required quantities of free poloxamine to undiluted fresh human serum or C1q-depleted serum (typical nanoparticles or polymer/serum volume, 1:4) in Eppendorf tubes (in triplicate) in a shaking water bath at $37^{\circ} \mathrm{C}$ for 30 $\mathrm{min}$. Reactions were terminated by the addition of PBS supplemented with $25 \mathrm{mM}$ EDTA. Control serum incubations contained saline (the same volume as nanoparticles or polymer) for assessing background levels of complement activation products. Zymosan, aggregated IgG, and mannan were used as positive control for alternative, classical, and lectin pathways, respectively. The level of the complement activation products was then measured by the respective ELISA kits and compared with control incubations in the absence of nanoparticles or polymer. In some experiments, nanoparticles/polymer-induced complement activation was monitored following pretreatment of serum with EGTA/Mg ${ }^{2+}(10 \mathrm{mM} / 2.5 \mathrm{mM})$, anti-MASP-2 monoclonal antibody (HyCult Biotechnology, The Netherlands), antiproperdin antibody (Quidel), an irrelevant murine lgG antibody (control), $\mathrm{N}$-acetylglucosamine (25 mM), and D-galactose (25 mM). Control serum incubations contained the same quantity of the added compounds, and nanoparticles/polymer was replaced with the same volume of saline. In all experiments $\mathrm{pH}$ was main tained between 6.8 and 7.1. To monitor the possible binding of complement activation products, we incubated nanospheres with standard samples of activation products. The level of the standard activation products in the supernatant was then measured by the respective ELISA test and compared with control incubations in the absence of nanospheres.

For quantification of complement activation products, standard curves were constructed using the assigned concentration of each respective standard supplied by the manufacturer and validated. The slope, intercept, and correlation coefficient of the derived best-fit line for $\mathrm{C} 4 \mathrm{~d}, \mathrm{Bb}, \mathrm{C} 5 \mathrm{a}$, and $\mathrm{SC} 5 \mathrm{~b}-9$ standard curves were within the manufacturers specified range. The efficacy of nanoparticles/polymer treatments was established by comparison with baseline levels using paired $t$ test, correlations between two variables were analyzed by linear regression, and differences between groups (when necessary) were examined using ANOVA followed by multiple comparisons with StudentNewmann-keuls test. Similar patterns were observed in all tested sera; the result of a typical experiment is presented.

Inductively Coupled Plasma Atomic Emission Spectrometry. Since operation of the alternative pathway is maintained or even accelerated by elevated $\mathrm{Mg}^{2+}$ levels, we monitored the contents of calcium and magnesium in poloxamine by inductively coupled plasma atomic emission spectrometry as described in detail else- where. ${ }^{22}$ The results confirmed negligible quantities of such cations (below $10 \mu \mathrm{g} / \mathrm{L}$ ), thus confirming complement activation via the alternative pathway is not driven by sample contamination with $\mathrm{Mg}^{2+}$.

Blood Concentration and Organ Distribution of Nanospheres. Radiolabeled nanospheres $\left(2 \times 10^{11}\right.$ particles) were injected intravenously into a group of male Wistar rats $(n=3)$ via the tail vain. Blood was removed at various intervals for determination of nanosphere concentration. Animals were killed at appropriate time intervals and distribution of nanoparticles in liver and spleen was determined. Full experimental details for radiolabeling and administration are presented elsewhere. ${ }^{6,26}$

Acknowledgment. We acknowledge financial support by the Danish Agency for Science, Technology and Innovation (Det Strategiske Forskningsråd, reference 09-065746/DSF and the European Commission FP7, reference CP-IP 212043-2 NAD.

\section{REFERENCES AND NOTES}

1. Moghimi, S. M.; Hunter, A. C.; Murray, J. C. Long-Circulating and Target-Specific Nanoparticles: Theory to Practice. Pharmacol. Rev. 2001, 53, 283-318.

2. Moghimi, S. M.; Szebeni, J. Stealth Liposomes and Long Circulating Nanoparticles. Critical Issues in Pharmacokinetics, Opsonization and Protein-Binding Properties. Prog. Lipid Res. 2003, 42, 463-478.

3. Moghimi, S. M.; Hunter, A. C.; Murray, J. C. Nanomedicine: Current Status and Future Prospects. FASEB J. 2005, 19, 311-330.

4. Stolnik, S.; Daudali, B.; Arien, A.; Whetstone, J.; Heald, C. R.; Garnett, M. C.; Davis, S. S.; lllum, L. The Effect of Surface Coverage and Conformation of Poly(ethylene oxide) (PEO) Chains of Poloxamer 407 on the Biological Fate of Model Colloidal Drug Carriers. Biochim. Biophys. Acta, Biomemb. 2001, 1514, 261-279.

5. Al-Hanbali, O.; Rutt, K. J.; Sarker, D. K.; Hunter, A. C.; Moghimi, S. M. Concentration Dependent Structural Ordering of Poloxamine 908 on Polystyrene Nanoparticles and their Modulatory Role on Complement Consumption. J. Nanosci. Nanotechnol. 2006, 6, 3126-3133.

6. Moghimi, S. M. Prolonging the Circulation Time and Modifying the Body Distribution of Intravenously Injected Polystyrene Nanospheres by Prior Intravenous Administration of Poloxamine 908. A Hepatic-Blockade Event or Manipulation of Nanosphere Surface In Vivo? Biochim. Biophys. Acta, Gen. Subj. 1997, 1336, 1-6.

7. Moghimi, S. M.; Hedeman, H.; Christy, N. M.; Illum, L.; Davis, S. S. Enhanced Hepatic-Clearance of Intravenously Administered Sterically Stabilized Microspheres in Zymosan-Stimulated Rats. J. Leukocyte Biol. 1993, 54, 513517.

8. Moghimi, S. M.; Hedeman, H.; muir, I. S.; Illum, L.; Davis, S. S. An Investigation of the Filtration Capacity and the Fate of Large Filtered Sterically-Stabilized Microspheres in Rat Spleen. Biochim. Biophys. Acta 1993, 1157, 233-240.

9. Moghimi, S. M. Mechanisms of Splenic Clearance of Blood Cells and Particles: Towards Development of New Splenotropic Agents. Adv. Drug Delivery Rev. 1995, 17, 103-115.

10. Ricklin, D.; Lambris, J. D. Complement-Targeted Therapeutics. Nat. Biotechnol. 2007, 25, 1265-1275.

11. Gros, P.; Milder, F. J.; Janssen, J. C. Complement Driven by Conformational Changes. Nat. Rev. Immunol. 2008, 8, 48-58.

12. Nilsson, B.; Nilsson Ekdahl, K.; Mollnes, T. E.; Lambris, J. D. The Role of Complement in Biomedical-Induced Inflammation. Mol. Immunol. 2007, 44, 82-94.

13. Andersen, A. J.; Hashemi, S. H.; Andresen, T. L.; Hunter, A. C.; Moghimi, S. M. Complement: Alive and Kicking Nanomedicines. J. Biomed. Nanotechnol. 2009, 5, 364-372.

14. Moghimi, S. M.; Andersen, A. J.; Hashemi, S. H.; Lettiero, B.; Ahmadvand, D.; Hunter, A. C.; Andresen, T. L.; Hamad, I.; Szebeni, J. Complement Activation Cascade Triggered by PEG-PL Engineered Nanomedicines and Carbon 
Nanotubes: The Challenges Ahead. J. Controlled Release 2010, 146, 175-181.

15. Markiewski, M. M.; Nilsson, B.; Ekdhal, K. N.; Mollnes, T. E.; Lambris, J. D. Complement and Coagulation: Strangers or Partners in Crime. Trend. Immunol. 2007, 28, 184-192.

16. Uziely, B.; Jeffers, S.; Isacson, R.; Kutsch, K.; Wei-Tsao, D.; Yehoshua, Z.; Libson, E.; Muggia, F. M.; Gabizon, A. Liposomal Doxorubicin: Activity and Unique Toxicities During Two Complementary Phase I Studies. J. Clin. Oncol. 1995, 13, 1777-1785.

17. Alberts, D. S.; Garcia, D. J. Safety Aspects of Pegylated Liposomal Doxorubicin in Patients with Cancer. Drugs (Suppl.) 1997, 54, 30-45.

18. Channan-Khan, A.; Szebeni, J.; Savay, S.; Liebes, L.; Rafique, N. M.; Alving, C. R.; Muggia, F. M. Complement Activation Following First Exposure to Pegylated Liposomal Doxorubicin (Doxil): Possible Role in Hypersensitivity Reactions. Annal. Oncology 2003, 14, 1430-1437.

19. Szebeni, J. Complement Activation-Related Pseudoallergy: A New Class of Drug-Induced Acute Immune Toxicity. Toxicology 2005, 216, 106-121.

20. Szebeni, J.; Baranyi, L.; Savay, S.; Bodo, M.; Milosevits, J.; Alving, C. R.; Bunger, R. Complement Activation-Related Cardiac Anaphylaxis in Pigs: Role of $\mathrm{C} 5$ a Anaphylatoxin and Adenosine in Liposome-Induced Abnormalities in ECG and Heart Function. Am. J. Physiol. Heart Cir. Physiol. 2006, 290, $\mathrm{H} 1050-\mathrm{H} 1058$.

21. Moghimi, S. M.; Hamad, I.; Andresen, T. L; Jørgensen, K.; Szebeni, J. Methylation of the Phosphate Oxygen Moiety of Phospholipid-Methoxy(polyethylene glycol) Conjugate Prevents PEGylated Liposome-Mediated Complement Activation and Anaphylatoxin Production. FASEB J. 2006, 20, 2591-2593.

22. Moghimi, S. M.; Hunter, A. C.; Dadswell, C. M.; Savay, S.; Alving, C. R.; Szebeni, J. Causative Factors Behind Poloxamer 188 (Pluronic F68, Flocor)-Induced Complement Activation in Human Sera. A Protective Role Against Poloxamer-mediated Complement Activation by Elevated Serum Lipoprotein Levels. Biochim. Biophys. ActaMol. Bas. Disease 2004, 1689, 103-113.

23. Moghimi, S. M.; Hunter, A. C. Complement Monitoring of Carbon Nanotubes. Nat. Nanotechnol. 2010, 5, 382.

24. Moghimi, S. M.; Hunter, A. C. Poloxamers and Poloxamines in Nanoparticle Engineering and Experimental Medicine. Trend. Biotechnol. 2000, 14, 411-420.

25. Blunk, T.; Luck, M.; Hochstrasser, D. F.; Sanchez, J. C.; Muller, B. W.; Muller, R. H. Kinetics of Plasma Protein Adsorption on Model Particles for Controlled Drug Delivery and Drug Targeting. Eur. J. Pharm. Biopharm. 1996, 42, 262-268.

26. Moghimi, S. M.; Pavey, K. D.; Hunter, A. C. Real-Time Evidence of Surface Modification at Polystyrene Lattices by Poloxamine 908 in the Presence of Serum: In Vivo Conversion of Macrophage-Prone Nanoparticles to Stealth Entities by Poloxamine 908. FEBS Lett. 2003, 547, 177-182.

27. Storm, G.; Belliot, S. O.; Daemen, T.; Lasic, D. D. Surface Modification of Nanoparticles to Oppose Uptake by the Mononuclear Phagocyte System. Adv. Drug. Delivery Rev. 1995, 17, 31-48.

28. Hamad, I. S.; Hunter, A. C.; Szebeni, J.; Moghimi, S. M. Poly(ethylene glycol)s Generate Complement Activation Products in Human Serum through Increased Alternative Pathway Turnover and a MASP-2-Dependent Process. Mol. Immunol. 2008, 26, 225-232.

29. Fujita, T. Evolution of Lectin-Complement Pathway and Its Role in Innate Immunity. Nat. Rev. Immunol. 2002, 2, 346-353.

30. Fujita, T.; Gigli, I.; Nussenzweig, V. Human C4-binding Protein. II. Role in Proteolysis of C4b by C3b Inactivator. J. Exp. Med. 1978, 148, 1044-1051.

31. Neal, J. C.; Stolnik, S.; Schacht, E.; Kenawy, E. R.; Garnett, M. C.; Davis, S. S.; Illum, L. In Vitro Displacement by Rat Serum of Adsorbed Radiolabeled Poloxamer and Poloxamine Copolymers from Model and Biodegradable Nanospheres. J. Pharm. Sci. 1998, 87, 1242-1248.
32. Andersson, J.; Bexborn, F.; Klinth, J.; Nilsson, B.; Ekdahl, K. N. Surface-Attached PEO in the Form of Activated Pluronic with Immobilized Factor H Reduces both Coagulation and Complement Activation in a WholeBlood Model. J. Biomed. Mat. Res. 2006, 76A, 25-34.

33. Toda, M.; Kitazawa, T.; Hirata, I.; Hirano, Y.; Iwata, H. Complement Activation on Surfaces Carrying Amino Groups. Biomaterials 2008, 29, 407-417.

34. Kimura, Y.; Miwa, T.; Zhou, L.; Song, W.-C. Activator-Specific Requirement of Properdin in the Initiation and Amplification of the Alternative Pathway Complement. Blood 2008, 111, 732-740.

35. Mangsbo, S. M.; Sanchez, J.; Anger, K.; Lamvris, J.; Ekdahl, K. N.; Loskog, A. S.; Nilsson, B.; Totterman, T. H. Complement Activation by $\mathrm{CpG}$ in a Human Whole Blood Loop System: Mechanisms and Immunomodulatory Effects. J. Immunol. 2009, 183, 6724-6732.

36. Schwaeble, W. J.; Reid, K. B. Does Properdin Crosslink the Cellular and the Humoral Immune Response. Immunol. Today 1999, 20, 17-21.

37. Wirthmueller, U.; Dewald, B.; Thelen, M.; Schafer, M. K.; Stover, C.; Whaley, K.; North, J.; Eggleton, P.; Reid, K. B.; Schwaeble, W. J. Properdin, a Positive Regulator of Complement Activation, Is Released from Secondary Granules of Stimulated Peripheral Blood Neutrophils. J. Immunol. 1997, 158, 4444-4451.

38. Peracchia, M. T.; Harnisch, S.; Pinto-Alphandary, H.; Gulik, A.; Dedieu, J. C.; Desmaele, D.; dAngelo, J.; Muller, R. H.; Couvreur, P. Visualization of in Vitro Protein-Rejecting Properties of PEGylated Stealth Polycyanoacrylate Nanoparticles. Biomaterials 1999, 20, 1269-1275.

39. Mosqueira, V. C. F.; legrand, P.; Gulik, A.; Bourdon, O.; Gref, R.; Labarre, D.; Barratt, G. Relationship between Complement Activation, Cellular Uptake and Surface Physicochemical Aspects of Novel PEG-Modified Nanocapsules. Biomaterials 2001, 22, 1967-2979.

40. Al-Hanbali, O.; Onwuzo, N. M.; Rutt, K. J.; Dadswell, C. M.; Moghimi, S. M.; Hunter, A. C. Modification of Stewards Biphasic Colorimetric Assay for Stable and Accurate Quantitative Determination of Pluronic and Tetronic Block Copolymers for Application in Biological Systems. Anal. Biochem. 2007, 361, 287-293. 